\title{
Evaluation of the AUXACOLOR system, a new method of clinical yeast identification
}

\author{
K G Davey, P M Chant, C S Downer, C K Campbell, D W Warnock
}

\begin{abstract}
Aims-To compare the AUXACOLOR yeast identification system with the API 20C system.

Methods-Yeast isolates $(n=215)$, comprising 16 species, were identified using the AUXACOLOR system and the API 20C system. Isolates that could not be identified with the API 20C system or which produced discrepant results in the two systems were identified by assimilation and fermentation procedures.

Results-AUXACOLOR correctly identified $150(85 \cdot 7 \%)$ of 175 germ tube negative isolates while API 20C identified 155 $(88 \cdot 6 \%)$. Incorrect identifications were more common with API 20C (7.4\%) than with AUXACOLOR (3.7\%). Of 110 isolates of four common pathogens (Candida glabrata, $C$ parapsilosis, $C$ tropicalis, and Cryptococcus neoformans), 82.7\% (91/110) were identified by AUXACOLOR while API 20C identified $74 \cdot 5 \%$ (82/110). Of 65 less common germ tube negative isolates, $55.4 \%(36 / 65)$ were identified by AUXACOLOR while API 20C identified $63 \cdot 1 \%$ (41/65).

Conclusion-Although it has a limited database of 26 species, the AUXACOLOR system is a useful method for identification of germ tube negative clinical yeast isolates. Compared with the API 20C, the AUXACOLOR system is simpler and quicker to set up, easier to interpret, and comparable in cost.

(f Clin Pathol 1995;48:807-809)
\end{abstract}

Keywords: AUXACOLOR, API 20C, yeasts.

The increasing incidence of opportunistic infections by yeasts has stimulated the development of both manual and automated commercial systems for identification of these pathogens. The new commercial systems are generally less time-consuming to set up, are simpler to interpret, and permit more rapid identification of isolates than the conventional methods which they have replaced..$^{1-6}$

The API 20C yeast identification system (bioMerieux, Marcy l'Etoile, France) provides reliable results in comparisons with classic identification methods. ${ }^{12}$ As a result, it has become the standard method for yeast identification in most clinical laboratories. Even though the system is faster than the classic assimilation and fermentation methods, ${ }^{2}$ the API $20 \mathrm{C}$ system is still time-consuming to set up, and the results are often difficult to interpret. The AUXA-
COLOR yeast identification system (Sanofi Diagnostics Pasteur, Marnes-la-Coquette, France) has been introduced recently to provide a simpler method of identifying a number of the more common yeast pathogens. Here, we report the results of a comparison of the AUXACOLOR system with the API 20C performed with a large number of clinical yeast isolates representing a wide range of species.

\section{Methods}

TEST ORGANISMS

A total of 215 yeast isolates, comprising 16 species, were tested. Of these, 194 were recent clinical isolates submitted to the Mycology Reference Laboratory (MRL), Bristol, for identification and 21 came from the National Collection of Pathogenic Fungi, held at the MRL, Bristol, or the culture collection of the Mycology Unit, Public Health Laboratory, Cardiff. The culture collection isolates were coded before being passed to the authors for testing. Their identities were unknown until the final results were examined. Isolates to be studied were subcultured onto Sabouraud dextrose agar plates and incubated at $37^{\circ} \mathrm{C}$ for at least 18 hours, but not more than 48 hours, before being tested by the two identification systems.

\section{AUXACOLOR SYSTEM}

The AUXACOLOR system consists of disposable plastic microplates containing 16 wells (figure). The first well is a negative control, while the second contains glucose and serves as a positive control. Twelve of the remaining 14 wells each contain a different sugar that may be assimilated by the test organism. Each sugar is dehydrated in the presence of a basic solution and a $\mathrm{pH}$ indicator, bromocresol purple. Growth in these wells results in turbidity and a colour change from blue to yellow. The final two wells contain a test for actidione resistance and a test for the detection of the phenoloxidase activity of Cryptococcus neoformans. The actidione resistance test well contains an oxido-reduction indicator and growth in this well results in a colour change from blue to pink or colourless. The development of a green or brown colour denotes a positive result in the phenoloxidase test well. All testing with the AUXACOLOR system was performed according to the manufacturer's instructions. Kits were stored at $4^{\circ} \mathrm{C}$ and were brought to room temperature before use.

The microplates were incubated at $30^{\circ} \mathrm{C}$ and read at 24, 48 and 72 hours. A five digit 


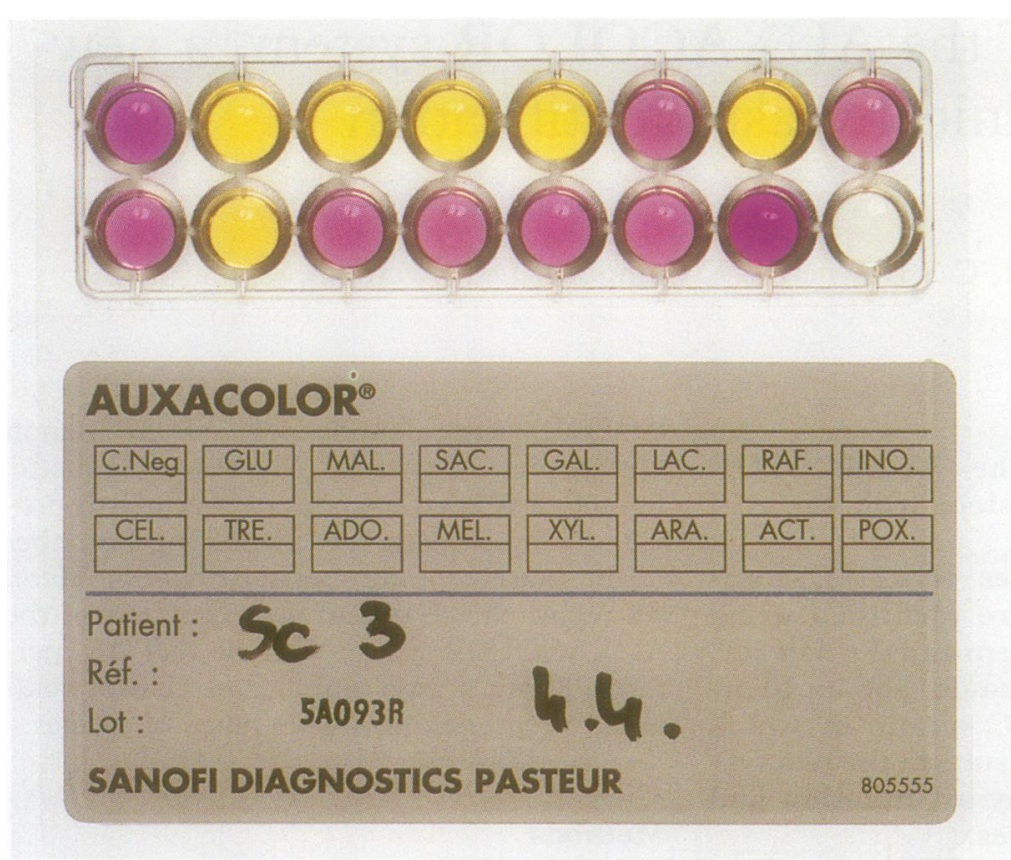

AUXACOLOUR profile of a $S$ cerevisiae isolate after incubation at $30^{\circ} \mathrm{C}$ for 48 hours.

profile number was generated for each isolate depending upon the reactions it produced. Two further digits were then added to the profile number depending on the growth characteristics of the isolate in morphological tests (see later). Identifications were made by referring to the list of numerical profiles provided by the manufacturer.

\section{API 20C SYSTEM}

The API 20C consists of a disposable plastic strip containing 20 cupules. The first cupule is a negative control, while the second contains glucose and serves as a positive control. The remaining 18 cupules each contain a different substrate that may be assimilated by the test organism. Tests with the API $20 \mathrm{C}$ system were performed according to the manufacturer's instructions. The results were recorded after 48 and 72 hours of incubation at $30^{\circ} \mathrm{C}$. A seven digit profile number was generated for each isolate depending upon the reactions it produced, which was then identified by referring to the APILAB computer program. Following the practice of most clinical laboratories processing routine specimens, we took the first organism listed under the profile number as the API 20C identification of the isolate, provided the profile had a $50 \%$ or greater chance of being correct.

MORPHOLOGICAL TESTS

Isolates were tested for germ tube formation in horse serum following incubation at $37^{\circ} \mathrm{C}$ for three hours. ${ }^{7}$ Isolates were inoculated onto cornmeal agar plates using the Dalmau method. ${ }^{8}$ The plates were incubated at $30^{\circ} \mathrm{C}$ and examined at 48, 72 and 96 hours for the presence of true hyphae, pseudohyphae, blastospores, chlamydospores, arthrospores, and capsules. India ink preparations were made to detect the presence of capsules.

\section{FURTHER TESTS}

Isolates that could not be identified with the API 20C system or which produced discrepant results in the API 20C and AUXACOLOR systems were identified by 35 traditional assimilation and fermentation procedures, utilising 29 different carbon sources. ${ }^{8}$

\section{Results}

A total of 215 yeast isolates were tested with both the API 20C and the AUXACOLOR yeast identification systems. The API 20C correctly identified $155(88.6 \%)$ of the 175 germ tube negative isolates, while the AUXACOLOR correctly identified $150(85 \cdot 7 \%)$ (table). Incorrect identifications were more common with the API 20C (16 strains, $7 \cdot 4 \%$ ) than with the AUXACOLOR (eight strains, $3.7 \%$ ) system. Of the 110 more common germ tube negative isolates (strains of Candida glabrata, $C$ parapsilosis, $C$ tropicalis, and $C$ neoformans), $82(74.5 \%)$ were correctly identified with API 20C, while the AUXACOLOR correctly identified 91 $(82 \cdot 7 \%)$. Of the 65 less common germ tube negative isolates tested, $41(63 \cdot 1 \%)$ were correctly identified with API 20C, while AUXACOLOR correctly identified 36 (55.4\%)

Only $23(57 \cdot 5 \%)$ of the $40 C$ albicans isolates tested were correctly identified with AUXA-

Identification of 215 yeast isolates with the AUXACOLOR and the API 20C systems

\begin{tabular}{|c|c|c|c|c|c|c|c|}
\hline \multirow[b]{2}{*}{ Organism } & \multirow[b]{2}{*}{$\begin{array}{l}\text { Total number } \\
\text { of isolates }\end{array}$} & \multicolumn{3}{|c|}{$A U X A C O L O R$} & \multicolumn{3}{|l|}{$A P I 20 C$} \\
\hline & & $\begin{array}{l}\text { Correctly } \\
\text { identified (n) }\end{array}$ & $\begin{array}{l}\text { Incorrectly } \\
\text { identified (n) }\end{array}$ & $\begin{array}{l}\text { Not } \\
\text { identified (n) }\end{array}$ & $\begin{array}{l}\text { Correctly } \\
\text { identified (n) }\end{array}$ & $\begin{array}{l}\text { Incorrectly } \\
\text { identified (n) }\end{array}$ & $\begin{array}{l}\text { Not } \\
\text { identified (n) }\end{array}$ \\
\hline Candida albicans & 40 & 23 & 1 & 16 & 32 & 3 & 5 \\
\hline Candida glabrata & 40 & 37 & 0 & 3 & 22 & 0 & 18 \\
\hline Candida guilliermondii & 4 & 3 & 0 & 1 & 3 & 1 & 0 \\
\hline Candida inconspicua & 4 & 4 & 0 & 0 & 2 & 0 & 2 \\
\hline Candida kefyr & 5 & 2 & 0 & 3 & 4 & 1 & 0 \\
\hline Candida krusei & 10 & 7 & 0 & 3 & 4 & 0 & 6 \\
\hline Candida lusitaniae & 6 & 3 & 2 & 1 & 4 & 0 & 2 \\
\hline Candida norvegensis & 4 & 0 & 2 & 2 & 0 & 2 & 2 \\
\hline Candida parapsilosis & 30 & 20 & 0 & 10 & 29 & 1 & 0 \\
\hline Candida tropicalis & 20 & 15 & 1 & 4 & 15 & 3 & 2 \\
\hline Cryptococcus albidus & 5 & 2 & 2 & $i$ & 5 & 0 & 0 \\
\hline Cryptococcus laurentii & 4 & 3 & 0 & i & 3 & 0 & 1 \\
\hline Cryptococcus neoformans & 20 & 19 & 0 & $\mathrm{i}$ & 16 & 2 & 2 \\
\hline Blastoschizomyces capitatus & 3 & 0 & 0 & 3 & 0 & 2 & 1 \\
\hline Saccharomyces cerevisiae & 10 & 4 & 0 & 6 & 9 & 0 & 1 \\
\hline Trichosporon beigelii & 10 & 8 & 0 & 2 & 7 & 1 & 2 \\
\hline
\end{tabular}


COLOR while the API $20 \mathrm{C}$ identified 32 $(80.0 \%)$. The major reason for this difference was that 10 of the 40 isolates tested failed to produce chlamydospores. Seven of these 10 chlamydospore negative isolates had profiles that were otherwise correct for $C$ albicans.

\section{Discussion}

In any evaluation of a new commercial identification system it is essential to perform independent identification of all isolates using an established procedure to act as an objective standard. With yeast identification this has, in the past, meant performing cumbersome and time-consuming assimilation and fermentation tests with 30 to 40 carbon sources. Not surprisingly, most recent comparisons have omitted this and instead have used one of the well established commercial systems, such as API 20C, as a reference standard. ${ }^{3-6910}$ Supplemental tests have been reserved for use where disagreement occurs between the method under evaluation and the reference method, or where the reference method fails to give an identification. ${ }^{5910}$ This approach was adopted in the present evaluation.

The AUXACOLOR system provides a simple and rapid method for the identification of the most commonly encountered yeasts in clinical practice. Our results suggest that this system is as reliable as the API 20C for the identification of $C$ neoformans, $C$ tropicalis and Trichosporon beigelii. The AUXACOLOR system proved more reliable than API 20C for the identification of $C$ krusei and $C$ glabrata, but less reliable for $C$ parapsilosis. The poor results for the identification of $C$ glabrata with the API 20C were because of the high proportion of isolates that failed to assimilate trehalose with this system, even after 72 hours of incubation.

In most laboratories identification of $C$ albicans isolates is accomplished using the serum germ tube test. Therefore, biochemical systems are seldom required for this organism. However, methods such as the API 20C and AUXACOLOR systems are useful for the iden- tification of germ tube negative strains of $C$ albicans, which can account for up to $5 \%$ of isolates of this species. Our results for the identification of $C$ albicans with the AUXACOLOR system were poor, but this was more often due to the failure of isolates to produce chlamydospores on the corn meal agar used than to the production of an incorrect biochemical profile. It must be emphasised that morphological observation remains essential to avoid errors in identification, no matter what system is used.

The AUXACOLOR system has a limited database of 26 species. This is smaller than the databases of other identification systems, but it includes the species usually encountered in clinical practice. Compared with API 20C, the AUXACOLOR system is quicker and simpler to set up, easier to read, and is comparable in cost. This system is recommended as an attractive alternative for the routine identification of germ tube negative isolates.

We thank Stuart Cranmer and Daniel Malait, Sanofi Diagnostics Pasteur, for supplying AUXACOLOR and for helpful discussion

1 Buesching WJ, Kurek K, Roberts GD. Evaluation of the modified API 20C system for identification of clinically important yeasts. $\mathcal{F}$ Clin Microbiol 1979;9:565-9.

2 Land GA, Harrison BA, Hulme KL, Cooper BH, Byrd JC. Evaluation of the new API $20 \mathrm{C}$ strip for yeast identification against a conventional method. $\mathcal{f}$ Clin Microbiol 1979;10: 357-64.

3 Salkin IF, Schadow KH, Bankaitis LA, McGinnis MR, Kemna ME. Evaluation of Abbott Quantum II yeast identification system. $\mathcal{F}$ Clin Microbiol 1985;22:442-4.

4 Salkin IF, Land GA, Hurd NJ, Goldson PR, McGinnis MR. Evaluation of YeastIdent and Uni-Yeast-Tek yeast identification systems. $\mathcal{F}$ Clin Microbiol 1987;25:624-7.

5 Pfaller MA, Preston T, Bale M, Koontz FP, Body BA Pfaller MA, Preston T, Bale M, Koontz FP, Body BA.
Comparison of the Quantum II, API Yeast Ident, and Comparison of the Quantum II, API Yeast Ident, and
AutoMicrobic systems for identification of clinical yeast AutoMicrobic systems for identification of
isolates. $\mathcal{F}$ Clin Micnobiol 1988;26:2054-8.

6 Fenn JP, Segal H, Barland B, Denton D, Whisenant J, Chun $\mathrm{H}$, et al. Comparison of updated Vitek yeast biochemical card and API 20C yeast identification systems. $\mathcal{f}$ Clin Microbiol 1994;32:1184-7.

7 Taschdjian CL, Burchall JJ, Kozinn PJ. Rapid identification of Candida albicans by filamentation on serum and serum substitutes. Am $\mathcal{F}$ Dis Child 1960;99:212-15.

8 Kreger-Van Rij NJW (ed). The yeasts. 3rd edn. Amsterdam: Elsevier Science Publishers, 1984.

9 El-Zaatari M, Pasarell L, McGinnis MR, Buckner J, Land GA, Salkin IF. Evaluation of the updated Vitek yeast identification data base. $\mathcal{F}$ Clin Microbiol 1990;28:1938-41.

10 St.-Germain G, Beauchesne D. Evaluation of the MicroScan rapid yeast identification panel. f Clin Microbiol 1991;29: 2296-9. 